\title{
Efeitos da reflexologia das mãos em idosos hospitalizados
}

\section{Efectos de la reflexología de las manos en los ancianos hospitalizados Effects of reflexology of the hands in the elderly hospitalized}

Fátima Helena do Espírito Santo ${ }^{1}$; Carla Lube de Pinho Chibante ${ }^{2}$; Matheus Cavalcante de Deus ${ }^{3}$;

Viviane de Souza Izidoro ${ }^{4}$; Camila dos Santos Lorena ${ }^{5}$

${ }^{1}$ Profa ${ }^{2}{ }^{a}$ da Escola de Enfermagem Aurora de Afonso Costa da Universidade Federal Fluminense,

Niterói, Rio de Janeiro $\backslash$ BR. Email: fatahelen@hotmail.com.

${ }^{2}$ Enfermeira. Doutoranda do curso de doutorado em Ciências do Cuidado em Saúde pela

Universidade Federal Fluminense/UFF. Email: carla-chibante@ig.com.br

${ }^{3}$ Acadêmico de Enfermagem e Licenciatura. $9^{\circ}$ Período. Escola de Enfermagem Aurora de Afonso Costa - EEAAC.

Universidade Federal Fluminense.UFF. Niterói - RJ. Email: mathdedeus@gmail.com

${ }^{4}$ Acadêmica de Enfermagem e Licenciatura. $8^{\circ}$ Período. Escola de Enfermagem Aurora de Afonso Costa - EEAAC.

Universidade Federal Fluminense. UFF. Niterói - RJ. Email: vivinesouza_izidoro@hotmail.com

${ }^{5}$ Acadêmica de Enfermagem e Licenciatura. $9^{\circ}$ Período. Escola de Enfermagem Aurora de Afonso Costa - EEAAC.

Universidade Federal Fluminense. UFF. Niterói - RJ. Email: camilalorena@id.uff.br.

Cómo citar este artículo en edición digital: Espirito Santo, F.H., Chibante, C.L.P., Deus, M.C. de., Izidoro, V.S. \& Lorena, C.S. (2016). Efeitos da reflexologia das mãos em idosos hospitalizados. Cultura de los Cuidados (Edición digital), 20(45).

Disponible en: < http:// dx.doi.org/10.14198/cuid.2016.45.16>

Correspondência: Carla Lube de Pinho Chibante. Rua Silveira Martins, 164/ Apto905, Catete, CEP: 2221000.

Rio de Janeiro (RJ).

Correo electrónico: carla-chibante@ig.com.br.

Recibido: 7/03//2015; Aceptado: 10/05/2016

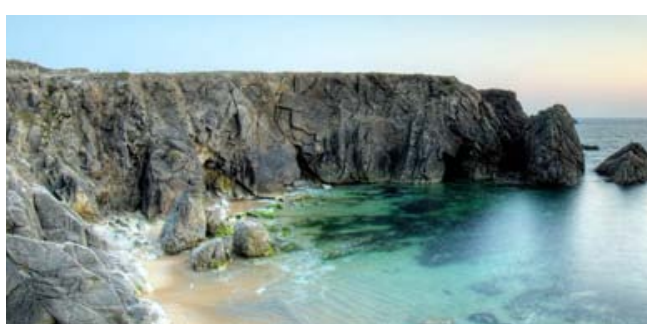

\section{ABSTRACT}

This research aimed to check the effects of reflexology of the hands in the elderly hospitalized. Consists of a clinical trial of type before and after, performed with 50 elderly inpatients in clinical medical and surgical wards of a university hospital. Three consultations were held of reflexology 48 hour interval being that 50 elderly made an consultation, 23 two consultations and 12 three consultations with control of the vital signs before and after each query, whose data were subjected to statistical analysis simple. The research project was approved by the Ethics Committee of the institution. The analysis of the data showed reduction in systolic blood pressure, diastolic blood pressure and heart rate in three consultations. Reflexology of the hands showed up as a practice that can be used as a complement to the nursing care, by promoting benefits the elderly hospitalized.

Keywords: Nursing; Massage; Complementary Therapies; Aged; Hospitalization.

\section{RESUMEN}

Este estudio tuvo como objetivo verificar la efectividad de la reflexología de las manos en los ancianos hospitalizados. Consiste en un ensayo clínico de tipo antes y después, realizado con 50 ancianos hospitalizados en clínicas médicas y quirúrgicas em las salas de un hospital universitário. Se celebraron tres consul- 
tas de reflexologia con intervalo de 48 horas resultando que 50 ancianos hicieron una consulta, 23 dos consultas y 12 tres consultas con el control de los signos vitales antes y después de cada consulta. Estos datos fueron sometidos a análisis estadístico simple. El proyecto de investigación fue aprobado por el Comité de ética de la institución. El análisis de los datos mostró reducción en la presión arterial sistólica, la presión arterial diastólica y pulso en tres consultas. Reflexología de las manos se mostró como una práctica que puede ser utilizada como un complemento a la atención de enfermería, mediante la promoción de beneficios de los ancianos hospitalizados.

Palabras clave: Enfermería; Massage; Terapias Complementarias; Anciano; Hospitalización.

\section{RESUMO}

Este estudo teve como objetivo verificar os efeitos da reflexologia das mãos em idosos hospitalizados. Consiste em um ensaio clínico do tipo antes e depois, realizado com 50 idosos internados nas enfermarias de clínica médica e cirúrgica de um hospital universitário. Foram realizadas três consultas de reflexologia com intervalo de 48 horas, sendo que 50 idosos fizeram uma consulta, 23 duas consultas e 12 três consultas, com controle dos sinais vitais, antes e após cada consulta, cujos dados foram submetidos à análise estatística simples. $\mathrm{O}$ projeto de pesquisa foi aprovado pelo Comitê de Ética da instituição. A análise dos dados evidenciou redução da pressão arterial sistólica, diastólica e da frequência cardíaca nas três consultas. A reflexologia das mãos mostrou-se como uma prática que pode ser utilizada como complementar ao cuidado de enfermagem, por promover benefícios ao idoso hospitalizado.

Palavras-chave: Enfermagem; Massagem; Terapias complementares; Idoso; Hospitalização.

\section{INTRODUÇÃO}

No Brasil, como em outros países em desenvolvimento, o aumento da população idosa é um fenômeno crescente. Entre 1980 e ano 2000 a população com mais de 60 anos cresceu $86,7 \%$. Projeções recentes indicam que esse segmento poderá ser responsável por quase $15 \%$ da população, ou seja, 14 milhões de idosos. Estima-se que este contingente se elevará a 31,8 milhões em 2025, alcançando a sexta posição em população com idade igual ou superior a 60 anos no Mundo (OMS, 2012; Chaimowicz, 2013).

Em geral, as doenças nos idosos são crônicas e múltiplas, perdurando por vários anos, exigindo avaliação contínua de uma equipe multiprofissional qualificada com preparo técnico e científico para avaliar, identificar e implementar ações de acordo com as necessidades e limitações inerentes ao processo de envelhecimento, as quais tendem a ser agravadas com a doença e hospitalização. Nesse contexto, cuidar do idoso implica em uma abordagem holística, que considera a enfermidade física como apenas uma das numerosas manifestações de um desequilíbrio básico do organismo em que a origem da doença e o seu desenvolvimento e cura envolvem a interação contínua da mente e do corpo (Capra, 2006, p. 127). Para isso, Capra (2006), discorre sobre o paradigma holístico e, ao propor novos rumos para a saúde, enfatiza a necessidade de se rever os atuais modelos de serviços, de instituições de ensino e de pesquisas em saúde. A transição para o novo modelo, entretanto, segundo o autor, precisa ocorrer de forma lenta e cuidadosa, por causa do enorme poder simbólico da terapia biomédica em nossa cultura ocidental.

Assim, é fundamental estimular o indivíduo a participar e entender a sua doença, a gestão de si mesmo. É preciso também ampliar seu processo de compreensão acerca da 
doença, da saúde, da vida e repensar as práticas assistenciais na perspectiva de outro olhar sobre o processo saúde-doença, para, desta maneira, desenvolver uma abordagem integral ao indivíduo (Souza e Vieira, 2005). Com isso, cabe ao enfermeiro o conhecimento e desenvolvimento de habilidades e estratégias para o cuidado integral do idoso hospitalizado, sendo as práticas não-farmacológicas potenciais aliadas. Essas práticas podem ser combinadas com práticas farmacológicas e incluem treinamentos físicos, técnicas de relaxamento e técnicas comportamentais (Eler e Jaques, 2006).

A combinação de intervenções não farmacológicas e farmacológicas proporcionam melhor controle da dor, menor consumo de analgésicos, redução da incidência de ansiedade e depressão, aumento da atividade e maior comprometimento da família com os cuidados (Morton et al, 2007). Dentre as práticas não farmacológicas este estudo aborda a reflexologia das mãos que é uma prática holística, que não isola uma doença para tratar seus sintomas, nem atua especificamente sobre um órgão ou sistema, mas na pessoa de forma integral visando promover um estado de equilíbrio e harmonia (Dougans e Ellis, 1998).

Esta técnica baseia-se no princípio de que existem áreas, ou pontos reflexos nos pés e nas mãos que correspondem a cada órgão, glândula e estrutura no corpo. Ao trabalhar nesses reflexos, reduz-se a tensão em todo o corpo permitindo a recuperação gradativa do bem-estar (Machado, 2008). Desta forma, a Reflexologia é aplicada como uma técnica de massagem em pontos existentes sobre as mãos ou pés, que representam todos os órgãos e os membros do corpo humano, promovendo relaxamento e equilíbrio corporal, porque, ao estimulá-los a energia corporal é produzida, é liberada e utilizada pelo organismo do paciente a um estado de equilíbrio ou homeostase, onde a circulação pode fluir desimpedida, os órgãos corporais, de fato agregações celulares, podem então voltar a um estado ou função normal (Kunz e Kunz,1997).

Portanto, trata-se de uma técnica específica de massagem aplicada a determinadas áreas, principalmente pés e mãos que permite alcançar o equilíbrio e gradativa recuperação do bem-estar e sua finalidade é corrigir os três fatores negativos implicados no processo saúde-doença que são: inflamação, congestão e tensão, pois os quadros inflamatórios e congestivos acarretam doenças e a tensão afeta o sistema imunológico (Albuquerque, 2011). A reflexologia tem sido muito usada como terapia complementar e sua popularidade está crescendo no mundo ocidental podendo ser utilizada com os mais diversos fins como: suporte ao tratamento do paciente oncológico para minimizar os efeitos da quimioterapia, dentre os quais: náusea, dor, diminuição do apetite e dificuldades de comunicação; além de melhorar a constipação crônica em crianças (Jiraingmongkol et al, 2002; Hodkinson, 2004; Bishop et al, 2003). À luz dessas considerações, este estudo tem como objetivo verificar os efeitos da reflexologia das mãos em idosos hospitalizados.

\section{TRAJETÓRIA METODOLÓGICA}

Foi realizado um ensaio clínico do tipo antes e depois, também chamado de ensaio clínico não-controlado, que descreve o curso da doença em um único grupo de pacientes antes e depois da exposição a uma intervenção. No caso desta pesquisa, a intervenção utilizada foi a reflexologia das mãos e buscou-se verificar os efeitos desta intervenção nos sinais vitais dos idosos hospitalizados (Fletcher e Fletcher, 2008). 
O estudo foi realizado nas enfermarias de clínica médica e cirúrgica de um hospital universitário localizado na região metropolitana do Estado do Rio de Janeiro no período de julho de 2012 a junho de 2013. A amostra foi constituída por 50 idosos, com idade igual ou superior a 60 anos, internados nas referidas clínicas. A seleção dos participantes teve como critérios de inclusão: idosos, a partir de 60 anos, com condições mentais preservadas. Foram excluídos da pesquisa idosos que solicitaram alta administrativa, transferidos para outras unidades de internação, ocorrência de óbito durante a internação, diagnóstico médico em prontuário de alguma doença infecto contagiosa e presença de acesso venoso em uma das mãos.

Após aprovação do projeto pelo Comitê de Ética da Instituição sob o n ${ }^{\circ}$ CAAEE: 0407.0.258.000-11, os participantes foram contatados nas referidas clínicas. Seguido da explicação detalhada do objetivo e método da pesquisa, foi solicitado a assinatura do Termo de Consentimento Livre e Esclarecido.

A coleta de dados ocorreu mediante aplicação de 3 sessões de reflexologia das mãos, com intervalo de 48 horas entre as mesmas. Antes e após cada sessão de reflexologia, os sinais vitais dos idosos eram verificados (pressão arterial sistólica, diastólica, frequência cardíaca e temperatura axilar). As sessões eram realizadas individualmente na própria enfermaria no leito dos idosos, por três dos pesquisadores com treinamento prévio da técnica. A duração de cada sessão foi em torno de 30 minutos. Participaram da primeira consulta de reflexologia 50 idosos, na segunda consulta 23 idosos e na terceira consulta 12 idosos.

Para análise dos dados foi elaborada uma planilha tipo excel com os nomes de cada par- ticipante em uma listagem que permitia fazer o acompanhamento individual de cada sessão. Ao final, os dados foram submetidos à análise estatística simples para identificação da prevalência dos efeitos da reflexologia nos sinais vitais do grupo estudado por meio de média aritmética e desvio padrão.

\section{RESULTADOS}

Dos 50 idosos participantes da pesquisa 20 (40\%) são do sexo feminino e 30 (60\%) do sexo masculino, com média de idade de 70,2 anos. Em relação ao estado civil, 31 (62\%) casados, 9 (18\%) viúvos, 7 (14\%) divorciados e 3 (6\%) solteiros.

Quanto à escolaridade, 4 (8\%) analfabetos, 23 (46\%) com ensino fundamental incompleto, 16 (32\%) com ensino fundamental completo, 6 (12\%) com ensino médio completo e 1 (2\%) com ensino superior completo. Em relação à profissão atual, 38 (76\%) aposentados, 8 (16\%) trabalham com carteira assinada, 2 (4\%) pensionistas e não tem emprego, respectivamente.

Após o contato inicial com os participantes, os mesmos foram orientados quanto à técnica de reflexologia das mãos e, posteriormente, a sessão era realizada com verificação dos sinais vitais (pressão arterial, temperatura axilar e frequência cardíaca) antes e após a massagem.

Dos 50 idosos participantes da pesquisa, 50 (100\%) realizaram uma sessão de reflexologia, 23 (46\%) duas sessões, e 12 (24\%) três sessões. As tabelas a seguir mostram as médias e desvios padrão dos sinais vitais verificados antes e depois das sessões de reflexologia.

As tabelas mostram uma redução nas médias da pressão arterial sistólica e diastólica, da frequência cardíaca e um aumento na média da temperatura axilar. 
Tabela 1- Diferença entre as médias da pressão sistólica, diastólica, frequência cardíaca e temperatura axilar antes e depois na $1^{\text {a }}$ consulta

$\mathbf{1}^{\mathrm{a}}$ Consulta $(\mathrm{n}=\mathbf{5 0})$

PA Sistólica

PA Diastólica

Frequência Cardíaca

Temperatura Axilar
Antes

Média \pm Desvio Padrão

$125,4 \pm 17,4$

$78,8 \pm 14,7$

$73,9 \pm 10,3$

$35,94 \pm 0,55$
Depois

Média \pm Desvio Padrão

$122,2 \pm 19,5$

$73,8 \pm 13,1$

$71,2 \pm 10,7$

$36,02 \pm 0,43$

Tabela 2- Diferença entre as médias da pressão sistólica, diastólica, frequência cardíaca e temperatura axilar antes e depois na $2^{\text {a }}$ consulta

\section{$2^{\text {a }}$ Consulta $(n=23)$}

PA Sistólica

PA Diastólica

Frequência Cardíaca

Temperatura Axilar
Antes

Média \pm Desvio Padrão

$127,4 \pm 16,8$

$83,9 \pm 11,2$

$77,3 \pm 10,4$

$35,89 \pm 0,48$
Depois

Média \pm Desvio Padrão

$120 \pm 16$

$79,1 \pm 13,8$

$74 \pm 11$

$36,04 \pm 0,46$

Tabela 3- Diferença entre as médias da pressão sistólica, diastólica, frequência cardíaca e temperatura axilar antes e depois na $3^{\text {a }}$ consulta

$3^{\text {a }}$ Consulta $(\mathbf{n}=12)$

\section{PA Sistólica}

PA Diastólica

Frequência Cardíaca

Temperatura Axilar
Antes

Média \pm Desvio Padrão

$138,3 \pm 25,5$

$89,8 \pm 13,4$

$84,3 \pm 10,8$

$35,91 \pm 0,50$

\author{
Depois \\ Média \pm Desvio Padrão \\ $125,8 \pm 25,7$ \\ $83,3 \pm 9,8$ \\ $79 \pm 10,4$ \\ $36,19 \pm 0,44$
}

\section{DISCUSSÃO}

Os resultados dessa pesquisa sugerem que a reflexologia das mãos reduz a pressão arterial, tanto sistólica quanto diastólica, e a frequência cardíaca na amostra estudada.

A alteração da pressão arterial após sessões de massagem foi relatada por um estudo realizado pela Touch Research Institute, onde foi comparado os efeitos entre sessões de massagem e relaxamento em pacientes com hipertensão arterial sistêmica. Os resultados mostraram que ambos os grupos tiveram redução dos níveis de ansiedade e dos níveis de depressão, porém apenas o grupo que recebeu a terapia da massagem apresentou diminuição na pressão sanguínea diastólica e sistólica, redução dos níveis de hormônio do estresse (cortisol) tanto na urina quanto na saliva e menor pontuação para depressão, ansiedade e hostilidade (Hernadez-Reif et al, 2000).

Com relação à frequência cardíaca, que nesse estudo apresentou diminuição da mesma nas três sessões de reflexologia, pesquisas que avaliaram a ação do toque terapêutico nos sinais vitais, verificaram influência na diminuição da frequência cardíaca, da ansiedade, melhoria do sono e das queixas de dor, além do aumento da confiança e diminuição da 
frequência respiratória (Ramada, Almeida e Cunha, 2013; Nascimento et al, 2012).

Outro estudo demonstrou que a massagem nos pés é capaz de melhorar a circulação sanguínea, promover o relaxamento e a capacidade de auto cura. Constatou-se que o toque estimula a secreção de endorfinas, além de reduzir a ansiedade e a dor. Também foi observado que a pulsação, a respiração e a pressão antes da massagem eram mais altas, proporcionando uma boa circulação, relaxamento e conforto (Jiraingmongkol et al, 2002).

A temperatura axilar teve aumento nas médias das três consultas de reflexologia nos idosos desse estudo. Esse aumento da temperatura axilar durante a massagem já foi evidenciado por outros autores que realizaram a reflexologia podal e o toque terapêutico (Bishop et al, 2003; Ramada, Almeida e Cunha, 2013).

Esse estudo corrobora o resultado de outra pesquisa que utilizou uma técnica com princípios semelhantes de imposição das mãos e canalização da energia, o Reiki. A referida pesquisa constatou que o fato de alterações biológicas poderem ser geradas dentro de um período curto de tempo demonstra o potencial desse recurso para aprimorar clinicamente fatores relevantes no estado de saúde das pessoas (Diaz-Rodriguez et al, 2011).

Considerando que existe uma contínua produção e troca de energia das células do corpo humano, quando esta é bloqueada por quaisquer motivos, instala-se um quadro de estagnação que impede seu fluxo aos músculos, órgãos e glândulas, diminuindo a capacidade deles e podendo ocasionar uma doença. Por isso, ao massagear os pontos reflexos que estimula a liberação da energia para circular por todo o organismo de forma adequada, ocorre consequente sensação de equilíbrio e bem-estar no indivíduo e o organismo em equilíbrio consegue reagir às outras formas de desequilíbrio orgânico, sejam eles estresse ou doenças (TASHIRO et al, 2001).

\section{CONCLUSÃO}

Ainda são pouco os estudos existentes sobre a reflexologia, entretanto os encontrados apontam que essa técnica apresenta algum efeito benéfico sobre o organismo, o que a torna uma terapia viável que requer investimentos acerca do seu uso como complementar ao cuidado em saúde. Além disso, nesse estudo os idosos, após as sessões de reflexologia, referiram uma sensação de bem-estar, relaxamento e até alívio de alguma dor ou sensação de mal-estar.

Tendo em vista que houve uma predominância de alterações dos padrões fisiológicos relacionados ao sistema circulatório, por meio da realização deste estudo, pode-se pensar em realizar essa técnica exclusivamente com pacientes hipertensos, devido aos benefícios encontrados e influência da reflexologia sobre os parâmetros do sistema circulatório.

Assim, na perspectiva holística de novos rumos na atenção à saúde do idoso, sugere-se que os enfermeiros e demais profissionais de saúde, busquem ampliar o olhar para além da visão tecnicista focada na doença para um olhar integral do indivíduo mediante utilização de práticas que favoreçam a melhoria da qualidade de vida das pessoas sob seus cuidados.

\section{REFERÊNCIAS:}

- Albuquerque, R.S. (2011). Reflexologia e Enfermagem. En L. Salles y M. Silva (Comps.), Enfermagem e as práticas complementares em saúde (pp. 175-188). São Caetano do Sul: Yendis Editora.

- Bishop, E., Mckinnon, E., Weir, E., Brown, D.W. (2003). Reflexology in the management of encopresis and chronic constipation. Pediatric nurs, 15(13), 20-21.

- Capra, F. (2006). O Ponto de mutação: a ciência a sociedade e a cultura emergente. São Paulo: Cultrix.

- Chaimowicz, F. (2013). Saúde do idoso. Belo Horizonte: 
Nescon UFMG.

- Diaz-Rodriguez, L., Arroyo-Morales, M., CantareroVillanueva, I., Férnandez-Lao, C., Polley, M. (2011). Uma sessão de Reiki em enfermeiras diagnosticadas com síndrome de Burnout tem efeitos benéficos sobre a concentração de IgA salivar e a pressão arterial. Rev latinoam enferm, 19(5), 1132-1138. Disponible em: http://www.scielo.br/pdf/rlae/v19n5/pt_10.pdf.

-Dougans, I., Ellis, S. (1998). Um guia passo a passo para a aplicação da reflexologia. São Paulo: Cultrix.

-Eler, G.J., Jaques, A.E. (2006). O enfermeiro e as terapias complementares para o alívio da dor. Arq ciências saúde UNIPAR, 10(3), 185-190.

- Fletcher, R.H., Fletcher, S.W. (2008). Epidemiologia clínica: elementos essenciais. Porto Alegre: Artmed.

- Hernadez-Reif, M., Field, T., Krasnegor, J., Theakston, H., Hossain, Z. \& Burman, I. (2000). High blood pressure and associated symptoms were reduced by massage therapy. J bodyw mov ther, 4(1), 31-38.

- Hodkinson, E. (2004). Complementary Therapies for palliative care. Therapy Directory. Disponible en: http:// www.therapy-directory.org.uk/content/palliative-care. html.

- Jiraingmongkol, P., Supatra, C., Phengchomjan, N., Bhanggananda, N. (2002). The effect of foot massage with biofeedback: A pilot study to enhance health promotion. Nurs health sci, 4(3), 1-10.

- Kunz, B., Kunz, K. (1997). Reflexologia: como restabelecer o equilíbrio energético. São Paulo: Editora Pensamento.

- Machado, N.M.S. (2008). Terapias de apoio aos servidores do Hospital Universitário de Santa Maria HUSM. Disponible em: http://www.husm.ufsm.br/ssst/pdf/ Reiki2008.pdf.
- Morton, P.G., Fontaine, D.K., Hudak, C.M., Gallo, B.M. (2007). Cuidados críticos de enfermagem: uma abordagem holística. Rio de Janeiro: Guanabara Koogan.

- Nascimento, L.B., Souza, V.P., Veras Filho, J., Araújo, E.C., Silva, T.C.L. (2012). Terapia integrativa e complementar em enfermagem: o toque terapêutico na Unidade de Terapia Intensiva. Rev enferm UFPE on line, 6(1), 9-16. Recuperado de http://www.revista. ufpe.br/revistaenfermagem/index.php/revista/article/ viewFile/1959/pdf_74.

- Nogueira, M.J.C. (1986). Abordagem holística: uma proposta para a enfermagem brasileira. $37^{\circ}$ Congresso Brasileiro de Enfermagem. Associação Brasileira de Enfermagem, Recife, Brasil.

- Organização Mundial da Saúde (OMS). (2012). Envejecimiento y salud. 65 ${ }^{\mathrm{a}}$ Asamblea Mundial de la Salud. Recuperado de http://apps.who.int/gb/ebwha/ pdf_files/WHA65-REC1/A65_REC1-sp.pdf.

- Ramada, N.C.O., Almeida, F.A., Cunha, M.L.R. (2013). Toque terapêutico: influência nos parâmetros vitais de recém-nascidos. Einstein (São Paulo), 11(4), 421-425. Recuperado de http://www.scielo.br/pdf/eins/v11n4/03. pdf.

- Souza, I.M.C., Vieira, A.L.S. (2005). Serviços públicos de saúde e medicina alternativa. Ciênc saúde coletiva (Online), 10(supl), 255-66. Recuperado de: http://www. scielo.br/scielo.php?script=sci_arttext $\&$ pid $=$ S141381232005000500026\&lng=en

- Tashiro, M.T.O., Orlandi, R., Martins, R.C.T., Santos, E. (2001). Novas Tendências Terapêuticas de Enfermagem - Terapias Naturais - Programa de Atendimento. Rev bras enferm (Online), 54(4), 658-67. Recuperado de: http://www.scielo.br/pdf/reben/v54n4/v54n4a15.pdf.



